Discussion There is a higher prevalence of poor sleep quality in SWNs compared to NSWNs. OH should be aware of any form of shiftwork as an important risk factor for poor sleep, as well as Trust managers acknowledging this workplace hazard. $\mathrm{OH}$ can provide staff with support for good sleep practice which aims to lead to healthier nurses.

\section{STRATEGIES OF TIME MANAGEMENT IN THE DAILY ROUTINE OF MEDICAL RESIDENTS OF INTERNAL MEDICINE IN A PUBLIC UNIVERSITY HOSPITAL}

Rafael Augusto Tamasauskas Torres*, Frida Marina Fischer. Department of Environmental Health, School of Public Health, University of São Paulo

\subsection{6/oemed-2018-ICOHabstracts. 1392}

Introduction Becoming a doctor is a complex process that usually consolidates during medical residency. This phase includes full immersion in professional activities with long working and studying hours. Junior doctors take care of patients in complex and difficult-to-manage care situations, both for their severity and institutional shortcomings. Although medical residency is recognised as an efficient form of professional training, there are several criticisms about work overload and long working hours.

Objective To identify strategies of time management in the daily routine of medical residents of Internal Medicine in a university hospital in São Paulo, Brazil.

Method In-depth interviews were conducted with eight randomly selected residents about their daily lives during the first year of residence.

Results The mean age was $25 \mathrm{y}$, being 4 men. Average hours of residence are $60 \mathrm{~h} /$ week. This excludes night shifts and work performed during days off. Over 8 months there is a 12 hour weekly night shift; and during 2 months there are 2 to 3 shifts per week. The reported highlights were: little time to accomplish domestic activities and commuting time to and from the hospital/home. Reduced time for leisure, sleep and study activities. Six out of eight participants reported activities during night shifts for supplementation of income. Some strategies for managing time were: moving to places close to the hospital, hiring cleaning ladies, eating out at the hospital restaurant, keeping a social circle restricted to other residents. The need for income supplementation was reported as a time management constraint.

Discussion Time management strategies during residence include measures to save time such as hiring third persons to perform domestic activities, and living close to the hospital. Some of the reported consequences are: the reduction of time to study, the restriction in the social relations and working odd-hours/days to supplement their income.

\section{URINARY 8-HYDROXYDEOXYGUANOSINE LEVELS OF EMERGENCY MEDICAL SHIFT WORKERS ARE HIGHER THAN THOSE OF FACTORY WORKERS}

Shintaro Watanabe*, Kazuaki Kawai, Yunshan Li. Department of Environmental Oncology Institute of Industrial Ecological Sciences, University of Occupational and Environmental Health, Japan

10.1136/oemed-2018-ICOHabstracts. 1393
Introduction Shiftwork is known as a risk factor of breast cancer and prostate cancer. In fact, the International Agency for Research on Cancer (IARC) classified shiftwork as Class 2A. One mechanism is that shiftwork increases cellular oxidative stress and results in DNA damage. Urinary 8-hydroxydeoxyguanosine $(8-\mathrm{OHdG})$ is frequently analysed as an oxidative stress marker. However, there have been few studies investigating the association of shiftwork with urinary 8-OHdG levels. In this study, we investigated the association between shiftwork with oxidative stress and urinary 8-OHdG levels.

Methods Urine samples from eight medical shift workers were collected before and after the night shift. The 8-OHdG levels were measured with a high-performance liquid chromatography (HPLC) system equipped with an electrochemical detector.

Results The mean level of urinary $8-\mathrm{OHdG}$ ( $\mu \mathrm{g} / \mathrm{ng}$ creatinine) in this study was $5.40 \pm 1.70$, and was higher than that of factory workers in a past study. There were no significant differences in the urinary $8-\mathrm{OHdG}$ levels before and after the night shift work.

Conclusion These results suggest that shiftwork, strain, and chronic fatigue lead to oxidative stress and DNA damage. The urinary $8-\mathrm{OHdG}$ level may be a useful biomarker, as an evaluating factor for chronic fatigue in regard to shiftwork.

\section{ASSOCIATION OF SHIFT-WORK, DAYTIME NAPPING, AND NIGHTTIME SLEEP WITH CANCER INCIDENCE AND CANCER-CAUSED MORTALITY IN DONGFENG-TONGJI COHORT STUDY}

${ }^{1}$ Yansen Bai ${ }^{*}$, 'Xiaoliang Li, 'Ke Wang, 'Shi Chen, 'Suhan Wang, 'Zhuowang Chen, ${ }^{1}$ Xiulong Wu, 'Wenshan Fu, 'Sheng Wei, 'Jing Yuan, 'Ping Yao, 'Xiaoping Miao, ${ }^{1}$ Xiaomin Zhang, ${ }^{1}$ Meian $\mathrm{He},{ }^{2}$ Handong Yang, ${ }^{1}$ Tangchun Wu, ${ }^{1}$ Huan Guo. ${ }^{1}$ Department of Occupational and Environmental Health, Tongji Medical College, Wuhan, China; ${ }^{2}$ Dongfeng Motor Corporation and Hubei University of Medicine, Shiyan, China

\subsection{6/oemed-2018-ICOHabstracts.1394}

Introduction More than 30\% of cancer deaths could be prevented by modifying or avoiding risk factors, such as unhealthy living habits, including night-shift work, daytime napping, and nighttime sleep. We aimed to investigate the independent and combined effects of these three sleep habits on cancer incidence and cancer-caused mortality among middle-aged and older Chinese in the Dongfeng-Tongji Cohort Study (27 009 retired workers were recruited from Dong-Feng Motor Corporation (DMC) in 2008, and 25978 participants were successfully followed-up during the first follow-up period from June, 2013 to October, 2013). During 114162 person-years of follow up, we identified a total of 1251 cancer cases and 379 cancer-caused deaths.

Methods Information on sleep habits, cancer incidences and mortalities were collected at baseline 2008. Self-reported cancer incidences and cancer-caused deaths were confirmed from DMC's health-care service system, which consists of five DMC-owned hospitals that covers all retired employees. Cox proportional hazards models were used to calculate the adjusted hazard ratios and 95\% confidence intervals (HRs, 95\% CIs).

Results Males experienced $\geq 20$ years of night-shift work, or no daytime napping had increased cancer incidence, when compared to males who did not have night-shift work or had daytime napping for $1 \sim 30 \mathrm{~min} \quad[\mathrm{HR}(95 \% \mathrm{CI})=1.27(1.01-$ 